\title{
Active Rectifier Control for Selective Fuse Tripping in a DC Microgrid
}

\author{
Jiajun Yang, Giampaolo Buticchi, Chunyang Gu \\ Key Laboratory of More Electric Aircraft Technology \\ of Zhejiang Province \\ University of Nottingham Ningbo China \\ Ningbo, China \\ jiajun.yang@nottingham.edu.cn
}

\author{
Pat Wheeler \\ University of Nottingham \\ Nottingham, UK \\ pat.wheeler@nottingham.ac.uk
}

\author{
Sebastian Brüske \\ Maschinenfabrik Reinhausen \\ Regensburg, Germany \\ s.brueske@reinhausen.com
}

\begin{abstract}
Distributed energy resources and new loads such as fast charging stations for electric vehicles are often dc-based. By using low-voltage de microgrids to connect such systems to the grid, conversion stages can be omitted and higher efficiencies can be achieved. Challenges exist with respect to the protection of dc microgrids, where dc fuses are a viable solution but selective tripping must be ensured. The grid-forming converter must be able to supply the fault current in the case of a line-to-line short-circuit in a feeder without tripping due to over-current. To avoid expensive over-sizing, this paper proposes to use a virtual resistance in the control of the grid-forming converter to limit its output current. The range of the virtual resistance is discussed for normal grid operation and the effect of the virtual resistance on limiting the bus current during a short-circuit fault is discussed. In addition, the impedance models of the source and load systems are presented. Based on this, the influence of the virtual resistance on the system stability is analyzed. To validate the theoretical part, a switching model is simulated and the results show a good agreement with the theoretical analysis.
\end{abstract}

Index Terms-dc microgrid, fault protection, dc fuse, voltage source rectifier, droop control

\section{INTRODUCTION}

The emerging use of dc loads, such as dc fast charging stations for electric vehicles, supports the change from completely ac based grids to a mix of ac and dc distribution. In grid areas with a high number of dc loads, potentially in combination with battery energy and photovoltaic systems, low voltage (LV) dc microgrids offer higher efficiencies in comparison to ac solutions due to the reduced number of conversion stages [1]. Such grids require power electronics converters for dc grid-forming and interfacing with the upstream ac grid. A common solution is to use a voltage source rectifier (VSR) as ac interfacing device. Depending on the ac grid voltage level, the voltage rating of the VSR and the requirement to provide galvanic isolation, a distribution transformer is needed between ac grid and VSR. A promising solution in dc grid applications without additional distribution transformer is the solid-state transformer (SST) [2], which provides galvanic isolation and medium voltage connectivity at lower volume and reduced losses.

The advantages of dc distribution and the increasing availability of grid-forming dc solutions are driving the need to overcome challenges that still exist with respect to detection and tripping time as well as selectivity and coordination of $\mathrm{LV}$ dc protection [3]. Stating that the required performance is not achieved by traditional dc fuse-based protection schemes, it is proposed in [4] to implement dc protection based on communication and a combination of ac and fast electronicbased dc protection. However, solid-state circuit breakers are not readily available and costly. In [5] it is shown that dc fuses are viable for protection of radial dc grids and can be used as an intermediate, cheaper solution with lower steady-state losses. A minimum output capacitance of the grid-forming unit is required to provide adequate fault current for selective tripping of feeder fuses. Sympathetic fuse tripping of parallel feeders is avoided by limiting the input capacitance of the load converters.

For clearing short-circuit faults at feeder level, it is important to avoid tripping of the grid-forming converter. Especially in dc microgrid configurations with a single grid-forming converter this would lead to a complete bus outage. A simple but expensive solution is to over-size the current rating of the grid-forming converter to provide the required fault current. This work proposes a virtual resistance in the converter control to limit the converter current for achieving selective fuse tripping. The stability and influence of fault conditions such as fault resistance and dc voltage reserve are discussed for a dc microgrid that consists of a VSR as grid-forming converter and two dual-active bridge (DAB) load converters.

In Section II, the VSR control in normal operation is presented. Section III explains the current limiting control technique in case of a linie-to-line fault at the point of load. The parameters of the considered dc microgrid are given in Section IV and the stability is analyzed in Section V. Simulation results are shown in Section VI. Conclusions are finally drawn in Section VII.

\section{CONDITIONS FOR NORMAL OPERATION OF DROOP CONTROLLED GRID-FORMING VSR}

In this section, the consideration of parameters selected for normal operation of the droop controlled grid-forming VSR is elaborated, under the hypothesis that the system is always individually stable. An example of a dc microgrid with a single grid-forming VSR and DAB converters as loads is depicted in 
Fig. 1, where the impedance of bus cable and filter capacitor are shown.

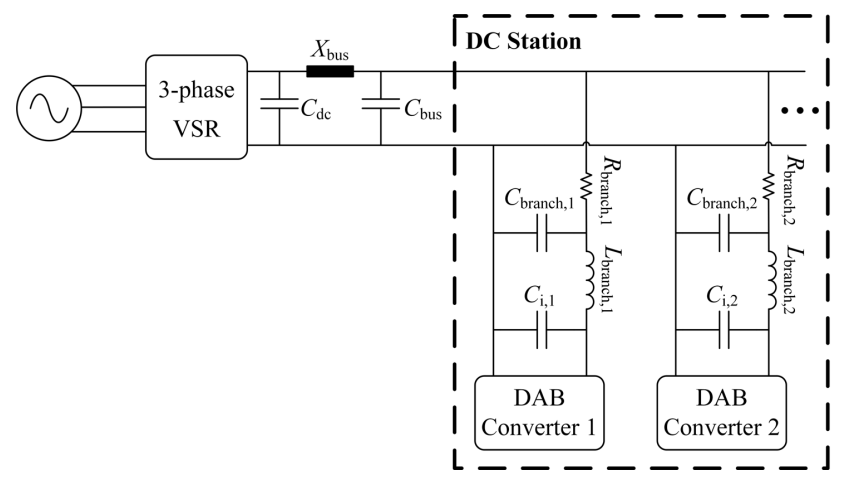

Fig. 1. Example of a dc microgrid with a single grid-forming converter

Fig. 2 shows the grid-forming VSR applied with droop control, where $V_{\mathrm{an}}, V_{\mathrm{bn}}$ and $V_{\mathrm{cn}}$ are the phase voltages of the grid, $I_{\mathrm{a}}, I_{\mathrm{b}}$ and $I_{\mathrm{c}}$ are the grid currents flowing into the VSR, $L_{\mathrm{a}}, L_{\mathrm{b}}$ and $L_{\mathrm{c}}$ are the filter inductances of the VSR, while $R_{\mathrm{a}}, R_{\mathrm{b}}$ and $R_{\mathrm{c}}$ are the filter resistances. $C_{\mathrm{dc}}$ is the output capacitor of the VSR, $V_{\mathrm{d}}$ and $V_{\mathrm{q}}$ are the output of the inner current controller, $V_{\mathrm{dc}}$ and $I_{\mathrm{o}}$ are the output voltage and output current of the VSR, $I_{\mathrm{dc}}$ is the output current of the VSR, $R_{\mathrm{v}}$ is the virtual resistor for droop control, and $\theta$ is the phase angle generated by the phase-locked loop (PLL). The active output power of the VSR is maximized by controlling the current on q-axis to be zero.

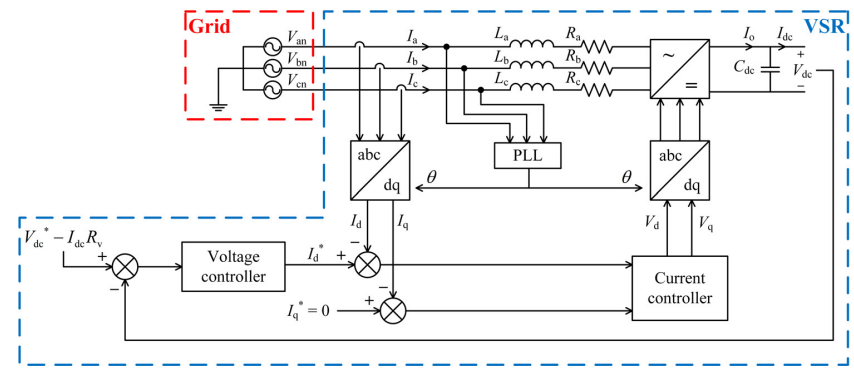

Fig. 2. Structure of a grid-forming VSR applied with droop control

Assuming that the grid is ideal, the grid voltage with positive sequence in d-q frame can be obtained as (1).

$$
\left[\begin{array}{l}
V_{\mathrm{gd}} \\
V_{\mathrm{gq}}
\end{array}\right]=\frac{2}{3}\left[\begin{array}{cc}
\cos \theta & -\sin \theta \\
\cos \left(\theta-\frac{2 \pi}{3}\right) & -\sin \left(\theta-\frac{2 \pi}{3}\right) \\
\cos \left(\theta+\frac{2 \pi}{3}\right) & -\sin \left(\theta+\frac{2 \pi}{3}\right)
\end{array}\right]^{\mathrm{T}}\left[\begin{array}{c}
V_{\mathrm{an}} \\
V_{\mathrm{bn}} \\
V_{\mathrm{cn}}
\end{array}\right]
$$

Hence, the open loop model of the grid-forming VSR in $\mathrm{d}-\mathrm{q}$ frame can be figured out as Fig. 3, where $\omega_{\mathrm{g}}$ is the fundamental frequency of the grid, $R_{\mathrm{f}}$ and $L_{\mathrm{f}}$ are the diagonal equivalent resistance and inductance of the $L$ filter in d-q frame, $D_{\mathrm{d}}$ and $D_{\mathrm{q}}$ are the corresponding duty cycle of $V_{\mathrm{d}}$ and $V_{\mathrm{q}}$.

By applying Kirchhoff's Voltage Law, the voltage equations can be obtained as (2).

$$
\left\{\begin{array}{l}
V_{\mathrm{d}}=-\left(R_{\mathrm{f}}+s L_{\mathrm{f}}\right) I_{\mathrm{d}}+\omega_{\mathrm{g}} L_{\mathrm{f}} I_{\mathrm{q}}+V_{\mathrm{gd}} \\
V_{\mathrm{q}}=-\left(R_{\mathrm{f}}+s L_{\mathrm{f}}\right) I_{\mathrm{q}}-\omega_{\mathrm{g}} L_{\mathrm{f}} I_{\mathrm{d}}+V_{\mathrm{gq}}
\end{array}\right.
$$

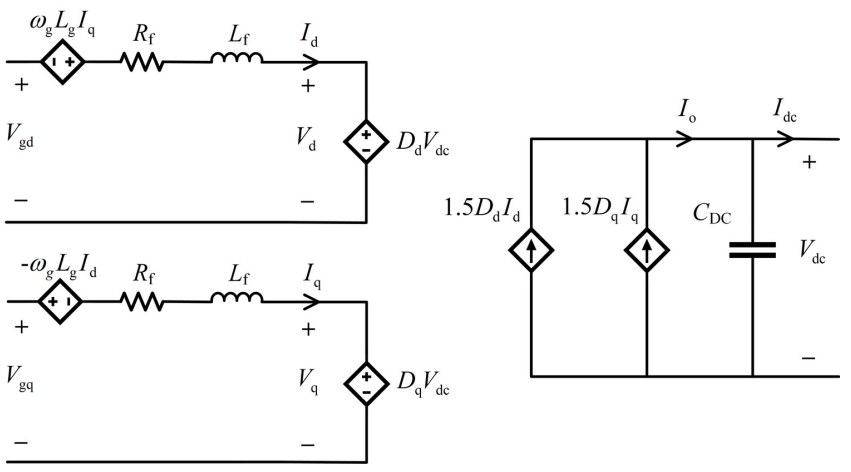

Fig. 3. The open loop model of grid-forming VSR in d-q frame

In steady state (i.e. $I_{\mathrm{q}}=0, I_{\mathrm{dc}}=I_{\mathrm{o}}$ ), the equation of active power can be simplified as (3).

$$
P=\left(V_{\mathrm{dc}}{ }^{*}-I_{\mathrm{dc}} R_{\mathrm{v}}\right) I_{\mathrm{dc}}=1.5 I_{\mathrm{d}} V_{\mathrm{d}}
$$

Hence, to make sure that $I_{\mathrm{dc}}$ has real solutions, or, the controller has constant output in steady state, the determinant of (3) needs to satisfy

$$
\Delta=\left(V_{\mathrm{dc}}{ }^{*}\right)^{2}-4 R_{\mathrm{v}} P \geq 0
$$

Then, the range of active power can be obtained as

$$
0 \leq P \leq \frac{\left(V_{\mathrm{dc}}{ }^{*}\right)^{2}}{4 R_{\mathrm{v}}}
$$

Besides, by replacing $V_{\mathrm{d}}$ in (3) with (2), the equation of active power can be expanded as

$$
P=-1.5 R_{\mathrm{f}}\left(I_{\mathrm{d}}-\frac{V_{\mathrm{gd}}}{2 R_{\mathrm{f}}}\right)^{2}+\frac{3 V_{\mathrm{gd}}^{2}}{8 R_{\mathrm{f}}}
$$

According to (6), the range of active power $P$ can be obtained as

$$
0 \leq P \leq \frac{3 V_{\mathrm{gd}}^{2}}{8 R_{\mathrm{f}}}
$$

Therefore, with the hypothesis that the grid-forming VSR is always stable regardless of its parameters, to make sure that it can work in the normal operation theoretically, or, the controllability of itself, it must satisfy

$$
0 \leq P \leq P_{\max }, \text { where } P_{\max }=\min \left(\frac{\left(V_{\mathrm{dc}}{ }^{*}\right)^{2}}{4 R_{\mathrm{v}}}, \frac{3{V_{\mathrm{gd}}}^{2}}{8 R_{\mathrm{f}}}\right)
$$

\section{Active Rectifier Control Based Protection TECHNIQUES FOR DC MICROGRID}

In this section, two control based protection techniques are proposed for dc microgrids. Fig. 4 shows a dc microgrid consisting of a grid-forming VSR and two DAB converters, where $V_{\mathrm{g}}$ is the grid voltage, $Z_{\mathrm{O}}$ is the dc-side output impedance of the grid-forming VSR, $Z_{\text {bus }}$ is the total impedance of filter capacitors and cables at the dc bus, $Z_{\mathrm{branch}, 1}$ and $Z_{\mathrm{branch}, 2}$ are the impedance of filter capacitors and cables at each feeder/branch. So, in steady state it exists 


$$
\begin{aligned}
& \left(I_{\mathrm{dc}}-I_{\mathrm{dc}, 2}\right) R_{\mathrm{branch}, 1}+\frac{P_{\mathrm{DAB}, 1}}{I_{\mathrm{dc}}-I_{\mathrm{dc}, 2}} \\
& =I_{\mathrm{dc}, 2} R_{\mathrm{branch}, 2}+\frac{P_{\mathrm{DAB}, 2}}{I_{\mathrm{dc}, 2}}
\end{aligned}
$$

where $P_{\mathrm{DAB}, 1}$ and $P_{\mathrm{DAB}, 2}$ is the power of the DAB converters, which behave as constant power loads. $R_{\text {branch }, 1}$ and $R_{\text {branch, } 2}$ are the cable resistances of each branch. Assuming that DAB converter 2 has a short circuit fault at one moment, the equation of the bus current becomes

$$
I_{\mathrm{dc}}=\frac{P_{\mathrm{DAB}, 1}+\left(I_{\mathrm{dc}}-I_{\mathrm{dc}, 2}\right)^{2} R_{\mathrm{branch}, 1}}{I_{\mathrm{dc}, 2}\left(R_{\mathrm{branch}, 2}+R_{\mathrm{short}}\right)}+I_{\mathrm{dc}, 2},
$$

where $R_{\text {short }}$ is the equivalent short-circuit resistance of DAB converter 2. Considering that $P_{\mathrm{DAB}, 1} \gg$ $\left(I_{\mathrm{dc}}-I_{\mathrm{dc}, 2}\right)^{2} R_{\mathrm{branch}, 1}$, the equation can be simplified as

$$
I_{\mathrm{dc}}=\frac{P_{\mathrm{DAB}, 1}}{I_{\mathrm{dc}, 2}\left(R_{\mathrm{branch}, 2}+R_{\mathrm{short}}\right)}+I_{\mathrm{dc}, 2}
$$

Deriving above equation yields

$$
\frac{\partial I_{\mathrm{dc}}}{\partial I_{\mathrm{dc}, 2}}=1-\frac{P_{\mathrm{DAB}, 1}}{I_{\mathrm{dc}, 2}{ }^{2}\left(R_{\mathrm{branch}, 2}+R_{\mathrm{short}}\right)}
$$

where it can easily be found

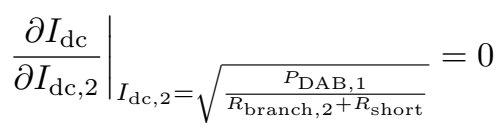

Hence, $I_{\mathrm{dc}}$ reaches minimum value when $I_{\mathrm{dc}, 2}=$ $\sqrt{\frac{P_{\mathrm{DAB}, 1}}{R_{\mathrm{branch}, 2}+R_{\text {short }}}}$. However, with the pre-conditions of the grid-forming VSR for normal operation, there are limits given by the grid voltage and the voltage utilization factor given by modulation method:

$$
\left\{\begin{array}{l}
\left(V_{\mathrm{dc}}\right)_{\min }=2 V_{\mathrm{gd}}, \text { if use SPWM } \\
\left(V_{\mathrm{dc}}\right)_{\min }=V_{\mathrm{gd}}, \text { if use SVPWM }
\end{array}\right.
$$

In this paper, Sinusoidal PWM (SPWM) is adopted. Therefore, the minimum power consumed on branch 2 can be given as

$$
P_{\text {short }, \min }=\frac{\left(2 V_{\mathrm{gd}}\right)^{2}}{R_{\mathrm{branch}, 2}+R_{\mathrm{short}}}
$$

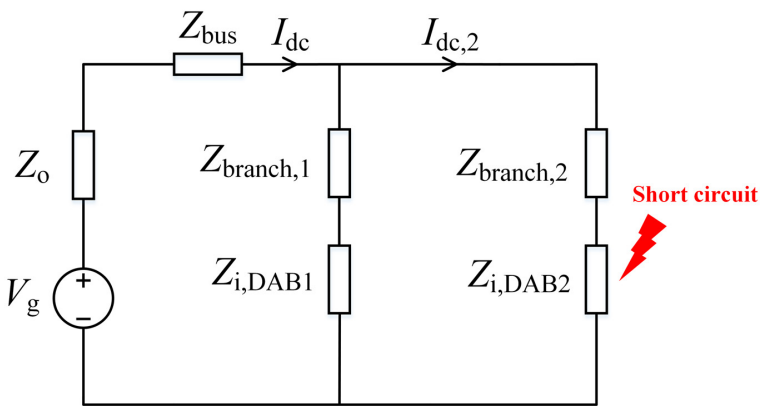

Fig. 4. The equivalent circuit of a dc microgrid consisting of a grid-forming VSR and two DAB converters
Considering that $P_{\text {short,min }}$ is larger than $P_{\mathrm{DAB}, 1}$, the minimum value of $I_{\mathrm{dc}, 2}$ can be calculated as

$$
\left(I_{\mathrm{dc}, 2}\right)_{\text {min }}=\sqrt{\frac{P_{\text {short }, \text { min }}}{R_{\text {branch }, 2}+R_{\text {short }}}}>\sqrt{\frac{P_{\mathrm{DAB}, 1}}{R_{\text {branch }, 2}+R_{\text {short }}}}
$$

And then, the minimum value of $I_{\mathrm{dc}}$ can be calculated as

$$
\left(I_{\mathrm{dc}}\right)_{\min }=\frac{P_{\mathrm{DAB}, 1}}{2 V_{\mathrm{gd}}}+\frac{2 V_{\mathrm{gd}}}{R_{\text {branch }, 2}+R_{\text {short }}}
$$

\section{A. droop Control Based Current Limiting Technique}

Generally, the droop control is used to achieve power sharing among the sources in multi-source systems. Yet, more than that, the droop control can be used to limit the bus current by choosing a proper virtual resistance value during the short-circuit fault, which can avoid tripping of the gridforming converter itself. When there is no fault, the system power is nearly constant regardless of $R_{\mathrm{v}}$. The value of $R_{\mathrm{v}}$ is constrained according to (5). The following inequality should always be kept true for real solutions of $I_{\mathrm{dc}}$ :

$$
R_{\mathrm{v}} \leq \frac{\left(V_{\mathrm{dc}}\right)^{2}}{4\left(P_{\mathrm{DAB}, 1}+P_{\mathrm{DAB}, 2}\right)}-R_{\mathrm{bus}}-R_{\text {branch }}
$$

Once the short-circuit fault occurs, the total power of the system is not constant anymore and can be influenced by $R_{\mathrm{v}}$. The effective range of the virtual resistance to limit the bus current during short circuit fault is given by

$$
R_{\mathrm{v}} \leq \frac{V_{\mathrm{dc}}{ }^{*}-2 V_{\mathrm{gd}}}{\left(I_{\mathrm{dc}}\right)_{\min }}
$$

\section{B. Modeling of Fuse Tripping}

Fuses are widely used in electrical power distribution system for protecting power electronics devices. Due to physical limitations e.g. self-heating of fuses that may prevent tripping in dc [6], the time constant must be considered when implemented in a dc microgrid. Fig. 5 shows the fuse model, where $I_{+}$and $I_{-}$are the bus currents and $I_{\mathrm{RMS}}$ is the rated RMS current and $a$ the pre-arc value of the fuse given by datasheet [7]. It is important to note that the proposed approach is limited by the physical constraints of the fuse and the bus current can be limited only within the range of fuse operation. If the operating conditions in terms of current rise, maximum current and maximum temperature are exceeded, the fuse may not be able to break the current.

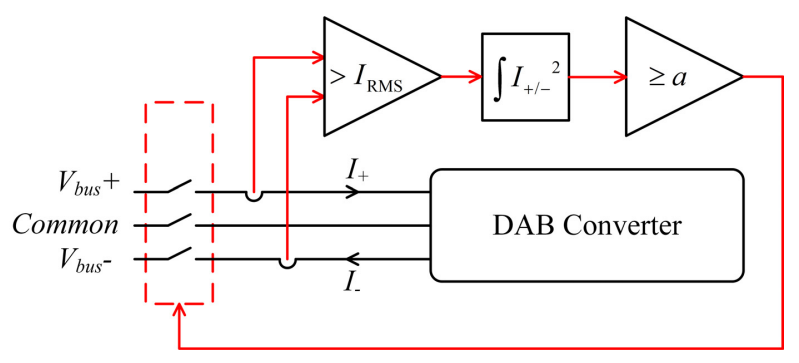

Fig. 5. Modeling of fuse tripping mechanism. 
TABLE I

PARAMETERS FOR SIMULATION

\begin{tabular}{ccc}
\hline \hline Symbol & Definition & Value \\
\hline$V_{\mathrm{g}}$ & Grid voltage (RMS) & $230 \mathrm{~V}$ \\
$R_{\mathrm{f}}$ & Filter resistance of VSR & $0.01 \Omega$ \\
$L_{\mathrm{f}}$ & Filter inductance of VSR & $100 \mu \mathrm{H}$ \\
$f_{\mathrm{VSR}}$ & Switching frequency of VSR & $10 \mathrm{kHz}$ \\
$G_{\mathrm{i}}$ & Current controller of VSR & $K_{\mathrm{p}}=0.2513, K_{\mathrm{i}}=25.1327$ \\
$G_{\mathrm{V}}$ & Voltage controller of VSR & $K_{\mathrm{p}}=0.205, K_{\mathrm{i}}=27.3278$ \\
$G_{\mathrm{f}}$ & Filter of droop control & $\mathrm{N} / \mathrm{A}$ \\
$V_{\mathrm{dc}}$ & DC bus voltage & $1500 \mathrm{~V}$ \\
$C_{\mathrm{dc}}$ & Output capacitance of VSR & $1 \mathrm{mF}$ \\
$I_{\mathrm{p}}$ & Over current protection value of VSR & $1000 \mathrm{~A}$ \\
$R_{\mathrm{bus}}$ & Resistance of DC bus cables & $0.02 \Omega$ \\
$L_{\mathrm{bus}}$ & Inductance of DC bus cables & $20 \mu \mathrm{H}$ \\
$C_{\mathrm{bus}}$ & Filter capacitance on DC bus & $0.5 \mathrm{mF}$ \\
$R_{\mathrm{branch}, 1 / 2}$ & Resistance of cables for branches & $0.0618 \Omega$ \\
$L_{\mathrm{branch}, 1 / 2}$ & Inductance of cables for branches & $0.0269 \mathrm{mH}$ \\
$C_{\mathrm{branch}, 1 / 2}$ & Parasitic capacitance of cables for branches & $0.204 \mu \mathrm{F}$ \\
$C_{\mathrm{i}, 1 / 2}$ & Input capacitance of DAB converter & $2 \mathrm{mF}$ \\
$f_{\mathrm{DAB}}$ & Switching frequency of DAB converter & $50 \mathrm{kHz}$ \\
$L_{\mathrm{lk}}$ & Leakage inductance of DAB converter & $10 \mu \mathrm{H}$ \\
$N$ & Turn ratio of transformer of DAB converter & 0.5 \\
$V_{\mathrm{o}}$ & Output voltage of DAB converter & $750 \mathrm{~V}$ \\
$C_{\mathrm{o}, 1 / 2}$ & Output capacitance of DAB converter & $2 \mathrm{mF}$ \\
$G_{\mathrm{v}, \mathrm{DAB}}$ & Voltage controller of DAB converter & $K_{\mathrm{p}}=0.02, K_{\mathrm{i}}=1$ \\
$P_{\mathrm{DAB}, 1 / 2}$ & Power of DAB converter & $150 \mathrm{~kW}$ \\
$R_{\mathrm{short}}$ & Short circuit resistance of DAB converter & $1 \Omega$ \\
$I_{\mathrm{RMS}}$ & Rated RMS current of virtual fuse [7] & $250 \mathrm{~A}$ \\
$\mathrm{a}$ & Pre-arc value of virtual fuse [7] & $21900 \mathrm{~A}{ }^{2} \mathrm{~s}$ \\
\hline \hline & & \\
\hline & &
\end{tabular}

\section{SPECIFICATIONS}

The parameters of the grid-forming VSR, dc bus capacitors, cables and DAB converters are listed in Table I. With these specifications, the limits of the virtual resistance can be calculated according to (19) and (18):

$$
R_{\mathrm{v}} \leq\left\{\begin{array}{l}
1.007, \text { for effective range of current limiting } \\
1.793, \text { for real solutions of } I_{\mathrm{dc}}
\end{array}\right.
$$

\section{The Influence OF ViRTual Resistance ON System STABILITY}

According to (20), it can be seen that the bus current can be reduced as much as possible by increasing $R_{\mathrm{v}}$ in a specific range. However, it is also necessary to check the influence of the virtual resistance on the system stability. In this section, the impedance model of the grid-forming VSR and $\mathrm{DAB}$ converters are derived, and then the system stability is analyzed.

\section{A. The DC Side Output Impedance of Grid-forming VSR}

The model of the grid-forming VSR is very similar to the model of a permanent magnet synchronous generator (PMSG), which has been derived and verified in [8]. Its individual stability has been analyzed in [9]. The main difference between the control of a PMSG and the grid-forming VSR is that the control is field-oriented or voltage-oriented. Since the PLL is nonlinear, and it is found that the dynamics of the phase angle caused by the PLL can influence the system impedance in dq frame [10], [11], the effect of the PLL is neglected in this paper. Moreover, if the droop control is considered, the transfer function block scheme will not change a lot, because the droop control only influences the dynamics of the transferred power but does not change the logical sequences of transfer functions. The transfer function block scheme of the grid-forming VSR with droop control can be derived as Fig. 6, where $G_{\mathrm{v}}(s)$ and $G_{\mathrm{i}}(s)$ are the transfer functions of the voltage controller and current controller, $G_{\mathrm{d}}(s)$ is the first-order time delay function with time constant of a switching period, $G_{\mathrm{f}}(s)$ is the filter added to the virtual resistor for droop control. As for the controller design, the 'symmetrical optimum' method in [12] is adopted. The detailed computation process is given in [8], [9].

Therefore, according to Fig. 6, the dc-side output impedance of the grid-forming VSR with droop control can be derived as (21). Fig. 7 shows the bode diagram of the output impedance of the grid-forming VSR with conventional control and droop control. Compared to conventional control, the bode diagram of droop control behaves as virtual resistor rather than an inductor at low frequencies.

\section{B. The Input Impedance of DAB Converter}

The input impedance of a DAB converter operating as constant power load has been derived and verified in [8], [13], given as (22), where $C_{\mathrm{i}}$ and $C_{\mathrm{o}}$ are the input capacitance and output capacitance, $G_{\mathrm{V}, \mathrm{DAB}}$ is the transfer function of the voltage controller, $R_{\text {load }}$ is the load resistance and $G_{1}, G_{2}$, $G_{3}$ and $G_{4}$ are the small signal gains.

\section{Stability Analysis}

Fig. 8 shows the Nyquist plot of the minor loop gain of the system and Fig. 9 shows the poles location of system. Both of them show that increasing the virtual resistance will make system tend to instability. Hence, there is a trade-off between current limiting and system stability when determining the virtual resistance.

$$
\begin{gathered}
Z_{\mathrm{o}}=\frac{G_{\mathrm{f}}(s) R_{\mathrm{v}} G_{\mathrm{v}}(s) G_{\mathrm{i}}(s)\left[1.5 V_{\mathrm{d}}-1.5\left(R_{\mathrm{f}}+s L_{\mathrm{f}}\right) I_{\mathrm{d}}\right]+\left(V_{\mathrm{dc}}{ }^{*}-I_{\mathrm{dc}} R_{\mathrm{v}}\right)\left[G_{\mathrm{i}}(s)+R_{\mathrm{f}}+s L_{\mathrm{f}}\right]}{\left[s C_{\mathrm{dc}}\left(V_{\mathrm{dc}}{ }^{*}-I_{\mathrm{dc}} R_{\mathrm{v}}\right)+G_{\mathrm{d}}(s) I_{\mathrm{o}}\right]\left[G_{\mathrm{i}}(s)+R_{\mathrm{f}}+s L_{\mathrm{f}}\right]+G_{\mathrm{d}}(s) G_{\mathrm{v}}(s) G_{\mathrm{i}}(s)\left[1.5 V_{\mathrm{d}}-1.5\left(R_{\mathrm{f}}+s L_{\mathrm{f}}\right) I_{\mathrm{d}}\right]} \\
Z_{\mathrm{i}, \mathrm{DAB}}=\frac{G_{3} G_{\mathrm{v}, \mathrm{DAB}} R_{\text {load }}+s C_{\mathrm{o}} R_{\text {load }}+1}{G_{4} R_{\text {load }}\left(G_{2}-G_{1} G_{\mathrm{v}, \mathrm{DAB}}\right)+s C_{\mathrm{i}}\left(G_{3} G_{\mathrm{v}, \mathrm{DAB}} R_{\text {load }}+s C_{\mathrm{o}} R_{\text {load }}+1\right)}
\end{gathered}
$$




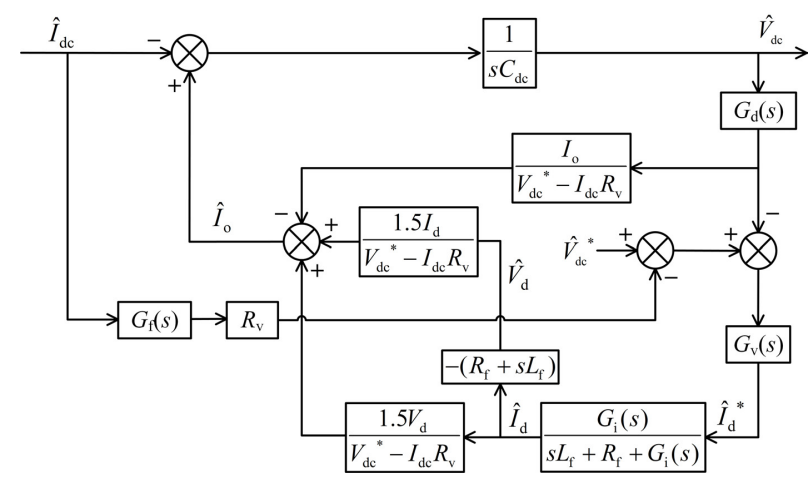

Fig. 6. Transfer function block scheme ofthe droop controlled grid-forming VSR.

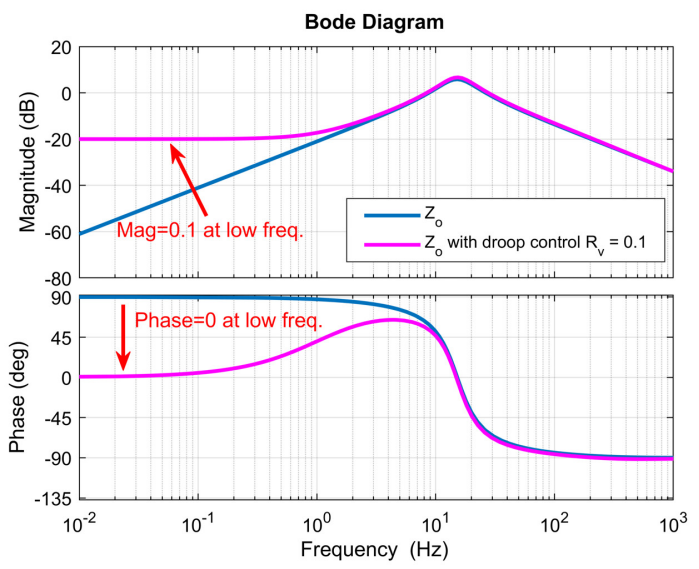

Fig. 7. Bode diagram of the output impedance of grid-forming VSR with conventional control and droop control.

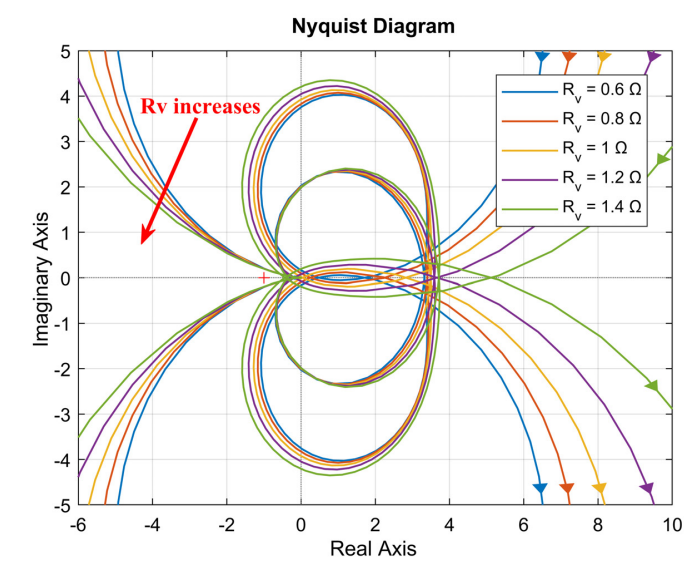

Fig. 8. Nyquist contour of minor loop gain of the system.

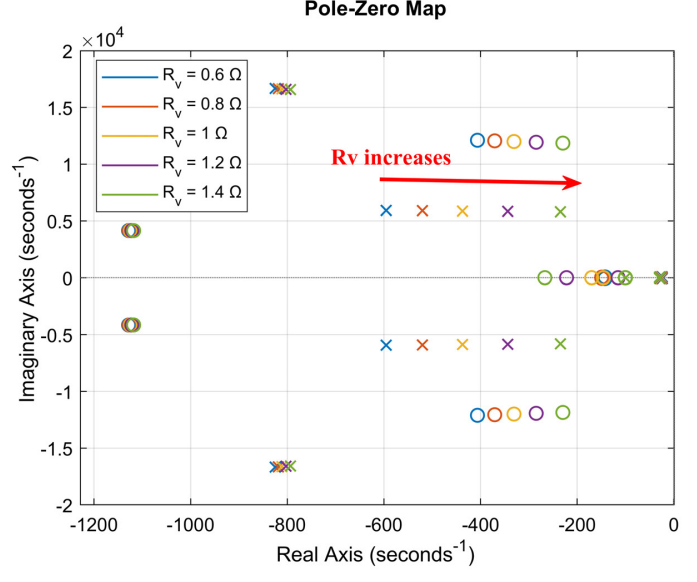

Fig. 9. Pole location of the system.
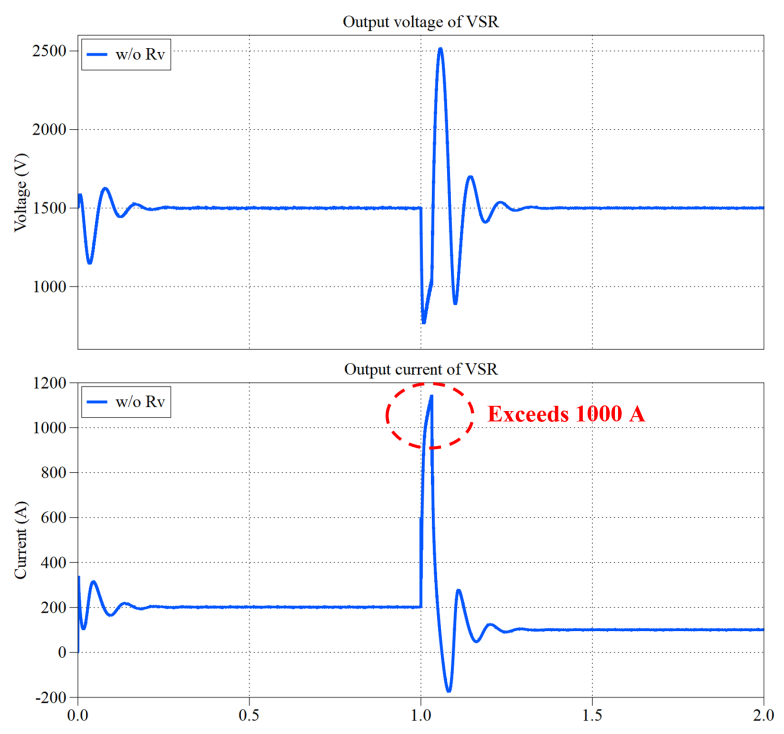

Fig. 10. Output voltage and output current of the VSR without droop control.

\section{Simulation Results}

In this section, the simulation results are given. Fig. 10 shows the output voltage and output current of the VSR without droop control. The short circuit happens at $t=1 \mathrm{~s}$. It can be seen that during the transients of the short-circuit fault, the output current goes beyond $1000 \mathrm{~A}$, meaning that the control of the VSR will be tripped off according to the specifications. Fig. 11 shows the output voltage and output current of the VSR with droop control. It can be seen that the output current is diminished lower than 1000 A during the transients of the short-circuit fault, avoiding the tripping of the VSR. Besides, it can be seen that before $t=1 \mathrm{~s}$, the larger the $R_{\mathrm{v}}$, the lower the output voltage and the higher the output current due to the constant power behavior of the system. After the short-circuit occurs, the system does not behave constant power anymore. It can be seen that the output current of the VSR can be reduced by increasing $R_{\mathrm{v}}$. In addition, the output current reaches minimum when $R_{\mathrm{v}}$ is equal or greater than 

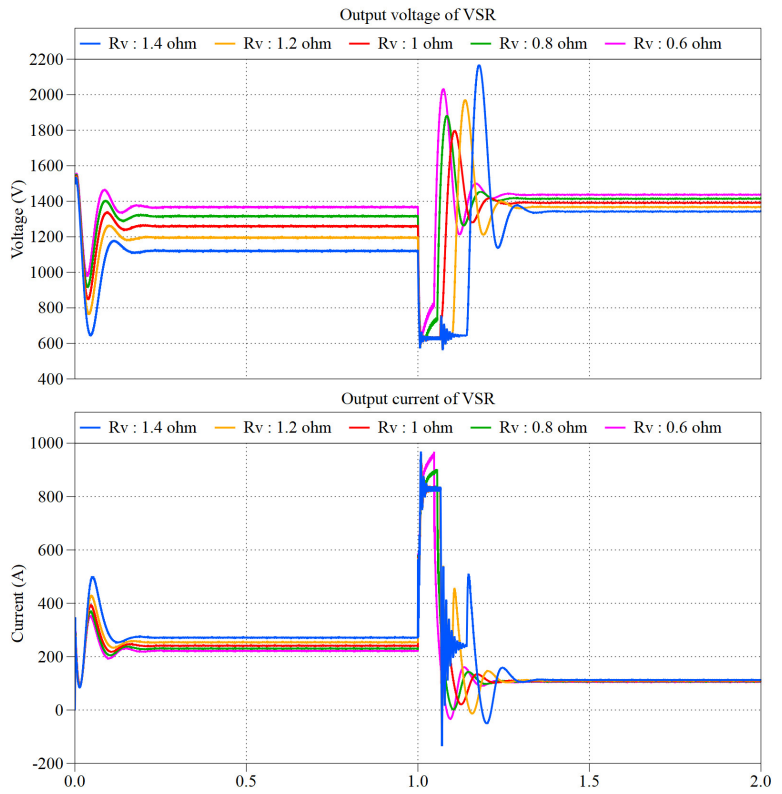

(a)
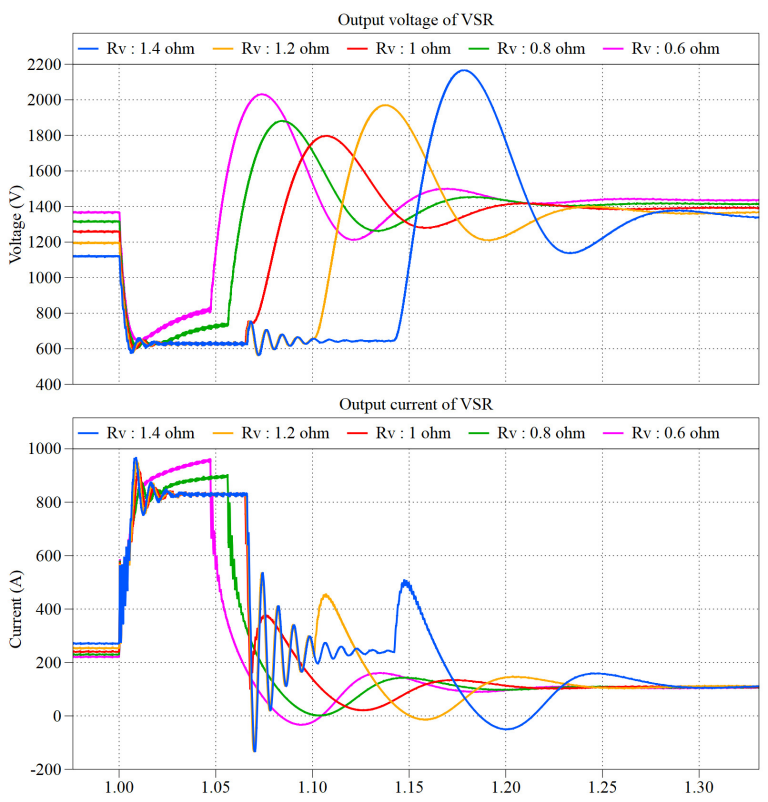

(b)

Fig. 11. Output voltage and output current of the VSR with droop control. (a) Full view and (b) Zoomed part.

$1 \Omega$, while the output voltage is clamped to $2 V_{\mathrm{gd}}$, which validates the effective range of current limiting in (20).

\section{CONCLUSION}

This paper investigates the active rectifier control based selective fuse tripping for a dc microgrid consisting of a gridforming VSR and two DAB converters. To ensure selective tripping in the case of a line-to-line short-circuit fault at the point of load, a virtual resistance in the droop control of the grid-forming converter is used to limit the bus current to avoid converter tripping due to over-current. The normal operation conditions of the droop controlled grid-forming VSR are derived. With these limitations, the range of the virtual resistance can be calculated. It shows that increasing the virtual resistance can effectively limit the bus current during the shortcircuit fault. In addition, the influence of the virtual resistance on the system stability is analyzed based on the impedance model of the grid-forming VSR and DAB converters. It can be seen that the system tends to instability when increasing the virtual resistance. Simulation results of the droop control based current limiting approach validate the calculated range of the virtual resistance.

\section{ACKNOWLEDGMENT}

This work is supported by the Key International Cooperation of National Natural Science Foundation of China under Grant 51920105011 .

\section{REFERENCES}

[1] H. Lotfi and A. Khodaei, "AC Versus DC Microgrid Planning," IEEE Trans. Smart Grid, vol. 8, no. 1, pp. 296-304, 2017.

[2] L. Ferreira Costa, G. De Carne, G. Buticchi, and M. Liserre, "The smart transformer: A solid-state transformer tailored to provide ancillary services to the distribution grid," IEEE Power Electron. Mag., vol. 4, no. 2, pp. 56-67, 2017.

[3] A. A. S. Emhemed, K. Fong, S. Fletcher, and G. M. Burt, "Validation of Fast and Selective Protection Scheme for an LVDC Distribution Network," IEEE Trans. Power Del., vol. 32, no. 3, pp. 1432-1440, 2017.

[4] A. A. Emhemed and G. M. Burt, "An advanced protection scheme for enabling an LVDC last mile distribution network," IEEE Trans. Smart Grid, vol. 5, pp. 2602-2609, 92014.

[5] S. Ravyts, G. V. D. Broeck, L. Hallemans, M. D. Vecchia, and J. Driesen, "Fuse-based short-circuit protection of converter controlled low-voltage dc grids," IEEE Trans. Power Electron., vol. 35, pp. 11694-11 706, 11 2020.

[6] D. Salomonsson, L. Söder, and A. Sannino, "Protection of low-voltage dc microgrids," IEEE Trans. Power Del., vol. 24, pp. 1045-1053, 2009.

[7] Eaton, Bussmann series: IEC High speed fuse links catalogue, 2020.

[8] J. Yang, H. Yan, C. Gu, S. Wang, W. Zhao, P. Wheeler, and G. Buticchi, "Modeling and Stability Enhancement of a Permanent Magnet Synchronous Generator Based DC System for More Electric Aircraft," IEEE Trans. Ind. Electron., pp. 1-1, 2021.

[9] J. Yang, G. Buticchi, C. Gu, and P. Wheeler, "Impedance-based Stability Analysis of Permanent Magnet Synchronous Generator for the More Electric Aircraft," in 2021 IEEE Workshop on Electrical Machines Design, Control and Diagnosis (WEMDCD), Apr. 2021, pp. 181-185.

[10] B. Wen, D. Dong, D. Boroyevich, R. Burgos, P. Mattavelli, and Z. Shen, "Impedance-Based Analysis of Grid-Synchronization Stability for Three-Phase Paralleled Converters," IEEE Trans. Power Electron., vol. 31, no. 1, pp. 26-38, Jan. 2016.

[11] L. Harnefors, M. Bongiorno, and S. Lundberg, "Input-Admittance Calculation and Shaping for Controlled Voltage-Source Converters," IEEE Trans. on Ind. Electron., vol. 54, no. 6, pp. 3323-3334, Dec. 2007.

[12] R. Teodorescu, M. Liserre, and P. Rodríguez, Grid Converters for Photovoltaic and Wind Power Systems. Chichester, UK: John Wiley \& Sons, Ltd, Jan. 2011.

[13] J. Yang, G. Buticchi, H. Yan, C. Gu, H. Zhang, and P. Wheeler, "Impedance-based Sensitivity Analysis of Dual Active Bridge DC-DC Converter," in 2019 IEEE 13th International Conference on Compatibility, Power Electronics and Power Engineering (CPE-POWERENG), Apr. 2019, pp. 1-5, iSSN: 2166-9546. 